\author{
A.E. Mirzakulova, N. Atakhan \\ Al-Farabi Kazakh National University, Almaty \\ (E-mail: aziza.mirzakulova@mail.ru)
}

\title{
Construction of the solution of the boundary value problem for integro differential equation with a small parameter in highest derivatives
}

\begin{abstract}
The article is devoted to the study analytical formula of solution of boundary value problem with initial jump for a linear integro-differential equation of $n+m$ order with a small parameter in the highest derivatives. In this paper singular perturbed homogeneous differential equation of $n+m$ order are constructed fundamental system of solutions. With the fundamental system of solutions are constructed Cauchy function and boundary functions. Using Cauchy function and boundary functions are obtained explicit analytical formula of solution of considered local boundary value problem for singular perturbed integro-differential equation of high order.
\end{abstract}

Key words: singularly perturbation, small parameter, boundary functions, Cauchy function, initial jump.

\section{Statement of the problem}

Consider the following integro differential equation with a small parameter in the highest derivatives

$$
L_{\varepsilon} y \equiv \sum_{r=1}^{m} \varepsilon^{r} A_{n+r}(t) \frac{d^{n+r} y}{d t^{n+r}}+\sum_{k=0}^{n} A_{k}(t) \frac{d^{k} y}{d t^{k}}=F(t)+\int_{0}^{1} \sum_{j=0}^{l-m+1} H_{j}(t, x) y^{(j)}(x, \varepsilon) d x
$$

with boundary conditions

$$
\left.h_{i} y \equiv \frac{d^{i} y}{d t^{i}}\right|_{t=0}=\alpha_{i}, \quad i=\overline{0, l-1},\left.\quad h_{l+i} y \equiv \frac{d^{i} y}{d t^{i}}\right|_{t=1}=\beta_{i}, \quad i=\overline{0, p-1}
$$

where $\varepsilon>0$ is a small parameter, $\alpha_{i}, \beta_{i}$ are known constants independent of $\varepsilon, A_{n+m}(t)=1, \quad m+n=l+p$.

Assume that following conditions hold:

I. $A_{i}(t) \in C^{n+m+1}([0,1]), i=\overline{0, n+m}, F(t) \in C([0,1])$ and functions $H_{j}(t, x) j=\overline{0, l-m+1}$ are defined in the domain $D=\{0 \leq t \leq 1,0 \leq x \leq 1\}$ and sufficiently smooth.

II. $A_{n}(t) \neq 0, \quad 0 \leq t \leq 1$;

III.The roots $\mu_{1} \neq \mu_{2} \neq \ldots \neq \mu_{m}$ of "additional characteristic equation" $\mu^{m}+A_{n+m-1}(t) \mu^{m-1}+\ldots+$ $+A_{n+1}(t) \mu+A_{n}(t)=0$ satisfy the following inequalities $\operatorname{Re} \mu_{1}<0, \operatorname{Re} \mu_{2}<0, \ldots, \operatorname{Re} \mu_{m}<0$ and $m<l$.

Similarly boundary value problem for ordinary differential equation was considered in [1]. In the particular case, similarly boundary value problem for singular perturbed integro-differential equation for this case $m=2$ and $l=2$ was considered in $[2,3]$.

\section{Construction of the fundamental system of solutions}

We consider the following homogeneous singularly perturbed equation associated with (1):

$$
L_{\varepsilon} y \equiv \sum_{r=1}^{m} \varepsilon^{r} A_{n+r}(t) \frac{d^{n+r} y}{d t^{n+r}}+\sum_{k=0}^{n} A_{k}(t) \frac{d^{k} y}{d t^{k}}=0 .
$$

The fundamental system of solutions of (3) in the interval $0 \leq t \leq 1$ has the following asymptotic representation as $\varepsilon \rightarrow 0$ [4]:

$$
y_{i}^{(q)}(t, \varepsilon)=y_{i 0}^{(q)}(t)+O(\varepsilon), \quad i=\overline{1, n}, \quad q=\overline{0, n+m-1}
$$




$$
y_{n+r}^{(q)}(t, \varepsilon)=\frac{1}{\varepsilon^{q}} \exp \left(\frac{1}{\varepsilon} \int_{0}^{t} \mu_{r}(x) d x\right)\left(\mu_{r}^{q}(t) y_{n+r, 0}(t)+O(\varepsilon)\right), \quad r=\overline{1, m}, \quad q=\overline{0, n+m-1},
$$

where functions $y_{i 0}(t), \quad i=\overline{1, n}$ are solutions of the problem

$$
\sum_{k=0}^{n} A_{k}(t) \frac{d^{k} y_{i 0}}{d t^{k}}=0, \quad y_{j 0}^{(i-1)}(0)=\delta_{i j}, \quad i=\overline{1, n}, \quad j=\overline{1, n},
$$

$\delta_{i j}$ is a Kronecker symbol and functions $y_{n+r, 0}(t), \quad r=\overline{1, m}$ are solutions of the problem

$$
p_{r}(t) \cdot y_{n+r, 0}^{\prime}(t)+q_{r}(t) \cdot y_{n+r, 0}(t)=0, \quad y_{n+r, 0}(0)=1, \quad r=\overline{1, m},
$$

where functions $p_{r}(t), q_{r}(t), r=\overline{1, m}$ are defined the following formula

$$
\begin{gathered}
p_{r}(t)=\sum_{i=0}^{m}(n+i) A_{n+i}(t) \mu_{r 0}^{n+i-1}(t), \quad r=\overline{1, m} ; \\
q_{r}(t)=\mu_{r 0}^{\prime}(t) \sum_{i=0}^{m} C_{n+i}^{2} A_{n+i}(t) \mu_{r 0}^{n+i-2}(t)+A_{n-1}(t) \mu_{r 0}^{n-1}(t), \quad r=\overline{1, m} ; \\
C_{n+i}^{2}=\frac{(n+i) !}{2 !(n+i-2) !}, \quad i=\overline{0, m} .
\end{gathered}
$$

\section{Construction of Cauchy function}

Definition 1. Function $K(t, s, \varepsilon)$ is called Cauchy function, if it is a solution of the problem

$$
\begin{gathered}
L_{\varepsilon} K(t, s, \varepsilon)=0, \quad t \neq s ; \\
K^{(j)}(s, s, \varepsilon)=0, \quad j=\overline{0, n+m-2}, \quad K^{n+m-1}(s, s, \varepsilon)=1,
\end{gathered}
$$

and Cauchy function can be represented as

$$
K(t, s, \varepsilon)=\frac{W(t, s, \varepsilon)}{W(s, \varepsilon)}
$$

where $W(s, \varepsilon)$ is the Wronskian of the fundamental system of solutions $y_{1}(s, \varepsilon), y_{2}(s, \varepsilon), \ldots, y_{n+m}(s, \varepsilon)$ of $(3)$, $W(t, s, \varepsilon)$ is the $n+m$-th order determinant obtained from the Wronskian $W(s, \varepsilon)$ by replacing the $n+m$-th row with $y_{1}(t, \varepsilon), y_{2}(t, \varepsilon), \ldots, y_{n+m}(t, \varepsilon)$.

\section{Construction of boundary functions}

Definition 2. Functions $\Phi_{i}(t, \varepsilon), \quad i=\overline{1, n+m}$ are called boundary functions for the boundary value problem (1) and (2), if they satisfy the following problem

$$
\begin{gathered}
L_{\varepsilon} \Phi_{i}(t, \varepsilon)=0, \quad i=\overline{1, n+m} \\
h_{k} \Phi_{i}(t, \varepsilon)=\delta_{k i}, \quad i=\overline{1, n+m}, \quad k=\overline{1, l+p},
\end{gathered}
$$

where $\delta_{k i}$ is a Kronecer symbol and boundary functions $\Phi_{i}(t, \varepsilon), \quad i=\overline{1, n+m}$ can be represented in the form

$$
\Phi_{i}(t, \varepsilon)=\frac{J_{i}(t, \varepsilon)}{J(\varepsilon)},
$$

where

$$
J(\varepsilon)=\left|\begin{array}{ccc}
h_{1} y_{1}(t, \varepsilon) & \ldots & h_{1} y_{n+m}(t, \varepsilon) \\
h_{2} y_{1}(t, \varepsilon) & \ldots & h_{2} y_{n+m}(t, \varepsilon) \\
\ldots & \ldots & \ldots \\
h_{l+p} y_{1}(t, \varepsilon) & \ldots & h_{l+p} y_{n+m}(t, \varepsilon)
\end{array}\right| .
$$

$J_{i}(t, \varepsilon)$ is the determinant obtained from $J(\varepsilon)$ by replacing the $i$-th row by the fundamental system of solutions $y_{1}(t, \varepsilon), y_{2}(t, \varepsilon), \ldots, y_{n+m}(t, \varepsilon)$ of $(3)$.

IV. $J(\varepsilon) \neq 0$. 


\section{Analytical formula of solution}

Denote by the right-hand side of equation (1)

$$
z(t, \varepsilon)=F(t)+\int_{0}^{1} \sum_{j=0}^{l-m+1} H_{j}(t, x) y^{(j)}(x, \varepsilon) d x .
$$

We seek the solution of differential equation $L_{\varepsilon} y=z(t, \varepsilon)$ in the form

$$
y(t, \varepsilon)=\sum_{i=1}^{n+m} C_{i} \Phi_{i}(t, \varepsilon)+\frac{1}{\varepsilon^{m}} \int_{0}^{t} K(t, s, \varepsilon) z(s, \varepsilon) d s
$$

where $\Phi_{i}(t, \varepsilon), \quad i=\overline{1, n+m}$ are boundary functions, $K(t, s, \varepsilon)$ is Cauchy function, $C_{i}, \quad i=\overline{1, n+m}$ are unknown constants, $z(t, \varepsilon)$ is an unknown function.

Substituting function (5) into equality (4), we obtain the following expression:

$$
\begin{aligned}
& z(t, \varepsilon)=F(t)+\int_{0}^{1} \sum_{j=0}^{l-m+1} H_{j}(t, x) \sum_{i=1}^{n+m} C_{i} \Phi_{i}^{(j)}(x, \varepsilon) d x+ \\
& +\int_{0}^{1} \sum_{j=0}^{l-m+1} H_{j}(t, x) \frac{1}{\varepsilon^{m}} \int_{0}^{t} K^{(j)}(t, s, \varepsilon) z(s, \varepsilon) d s d x .
\end{aligned}
$$

Replacing the order of sum and integral of equality (6), we obtain

$$
\begin{gathered}
z(t, \varepsilon)=F(t)+\sum_{i=1}^{n+m} C_{i} \int_{0}^{1} \sum_{j=0}^{l-m+1} H_{j}(t, x) \Phi_{i}^{(j)}(x, \varepsilon) d x+ \\
+\int_{0}^{1} z(s, \varepsilon) d s \frac{1}{\varepsilon^{m}} \int_{s}^{1} \sum_{j=0}^{l-m+1} H_{j}(t, x) K^{(j)}(t, s, \varepsilon) d x .
\end{gathered}
$$

Introducing of additional symbols of (7), we obtain the following Fredholm integral equation of the second kind:

$$
z(t, \varepsilon)=f(t, \varepsilon)+\int_{0}^{1} H(t, s, \varepsilon) z(s, \varepsilon) d s,
$$

where

$$
\begin{gathered}
f(t, \varepsilon)=F(t)+\sum_{i=1}^{n+m} C_{i} \int_{0}^{1} \sum_{j=0}^{l-m+1} H_{j}(t, x) \Phi_{i}^{(j)}(x, \varepsilon) d x \\
H(t, s, \varepsilon)=\frac{1}{\varepsilon^{m}} \int_{s}^{1} \sum_{j=0}^{l-m+1} H_{j}(t, x) K^{(j)}(x, s, \varepsilon) d x .
\end{gathered}
$$

V. 1 is not an eigenvalue of the kernel $H(t, s, \varepsilon)$.

In view of condition $\mathrm{V}$ integral equation (8) has an unique solution, that can be represented in the form

$$
z(t, \varepsilon)=f(t, \varepsilon)+\int_{0}^{1} R(t, s, \varepsilon) f(s, \varepsilon) d s,
$$

where $R(t, s, \varepsilon)$ is a resolvent of the kernel $H(t, s, \varepsilon)$. 
Substituting equality (9) into function (5), we obtain analytical formula of solution of boundary value problem (1) and (2):

$$
y(t, \varepsilon)=\sum_{i=1}^{n+m} C_{i} Q_{i}(t, \varepsilon)+P(t, \varepsilon),
$$

where $C_{i}, \quad i=\overline{1, n+m}$ are unknown constants, functions $Q_{i}(t, \varepsilon), \quad i=\overline{1, n+m}, \quad P(t, \varepsilon)$ can be represented in the form

$$
\begin{gathered}
Q_{i}(t, \varepsilon)=\Phi_{i}(t, \varepsilon)+\frac{1}{\varepsilon^{m}} \int_{0}^{t} K(t, s, \varepsilon) \bar{\varphi}_{i}(s, \varepsilon) d s, \quad i=\overline{1, n+m} ; \\
P(t, \varepsilon)=\frac{1}{\varepsilon^{m}} \int_{0}^{t} K(t, s, \varepsilon) \bar{F}(s, \varepsilon) d s,
\end{gathered}
$$

where $\Phi_{i}(t, \varepsilon), \quad i=\overline{1, n+m}$ are boundary functions, $K(t, s, \varepsilon)$ is Cauchy function, functions $\bar{\varphi}_{i}(s, \varepsilon)$, $i=\overline{1, n+m}, \bar{F}(s, \varepsilon)$ can be represented in the form

$$
\begin{gathered}
\bar{\varphi}_{i}(s, \varepsilon)=\int_{0}^{1} \sum_{j=0}^{l-m+1} \bar{H}_{j}(s, x, \varepsilon) \Phi_{i}^{(j)}(x, \varepsilon) d x, \quad i=\overline{1, n+m} \\
\bar{H}_{j}(s, x, \varepsilon)=H_{j}(s, x)+\int_{0}^{1} R(s, p, \varepsilon) H_{j}(p, x) d p, \quad j=\overline{0, l-m+1} \\
\bar{F}(s, \varepsilon)=F(s)+\int_{0}^{1} R(s, p, \varepsilon) F(p) d p .
\end{gathered}
$$

Using boundary conditions (2) into solution (10), we find constants $C_{i}=\alpha_{i-1}, \quad i=\overline{1, l}$ and for determining constants $C_{l+i}, \quad i=\overline{1, p}$, we need to solve the system of algebraic equation

$$
\left\{\begin{array}{l}
C_{l+1}\left(1+d_{l+1}(1, \varepsilon)\right)+C_{l+2} d_{l+2}(1, \varepsilon)+\ldots+C_{l+p} d_{l+p}(1, \varepsilon)=\beta_{0}-e(1, \varepsilon)-\sum_{i=1}^{l} \alpha_{i-1} d_{i}(1, \varepsilon) \\
\ldots \ldots \ldots \ldots \\
\left.C_{l+1} d_{l+1}^{(p-1)} 1, \varepsilon\right)+C_{l+2} d_{l+2}^{(p-1)}(1, \varepsilon)+\ldots+C_{l+p}\left(1+d_{l+p}^{(p-1)}(1, \varepsilon)\right)=\beta_{p-1}-e^{p-1}(1, \varepsilon)- \\
-\sum_{i=1}^{l} \alpha_{i-1} d_{i}^{(p-1)}(1, \varepsilon),
\end{array}\right.
$$

where

$$
\begin{gathered}
d_{i}^{(j)}(1, \varepsilon)=\frac{1}{\varepsilon^{m}} \int_{0}^{1} K^{(j)}(1, s, \varepsilon) \bar{\varphi}_{i}(s, \varepsilon) d s, \quad i=\overline{1, l+p}, \quad j=\overline{0, p-1} \\
e^{(j)}(1, \varepsilon)=\frac{1}{\varepsilon^{m}} \int_{0}^{1} K^{(j)}(1, s, \varepsilon) \bar{F}(s, \varepsilon) d s, \quad j=\overline{0, p-1} .
\end{gathered}
$$

Let the main determinant $\Delta(\varepsilon)=\bar{\Delta}+O(\varepsilon)$ of system (13).

VI. $\bar{\Delta} \neq 0$

Theorem. Let the conditions I-VI are valid. Then boundary value problem (1) and (2) on the interval $[0,1]$ has an unique solution, expressed by the formula

$$
y(t, \varepsilon)=\sum_{i=1}^{l} \alpha_{i-1} Q_{i}(t, \varepsilon)+\sum_{i=1}^{p} \bar{C}_{l+i} Q_{l+i}(t, \varepsilon)+P(t, \varepsilon),
$$

where $Q_{i}(t, \varepsilon), \quad i=\overline{1, n+m}, \quad P(t, \varepsilon)$ are defined by the formula (11),(12), $\bar{C}_{l+i}, i=\overline{1, p}$ are solutions of the system (13). 


\title{
References
}

1 Касымов К.А., Нургабыл Д.Н., Уаисов А.Б. Асимптотические оценки решения краевой задачи с начальным скачком для линейных дифференциальных уравнений с малым параметром при производных // Украинский. мат. журн. - 2013. - № 5. - С. 629-641.

2 Дауылбаев M.K., Мирзакулова А.Е. Краевые задачи с начальными скачками для сингулярно возмущенных интегро-дифференциальных уравнений // Нелинейные колебания. - 2016. - Т. 19. - № 1 . - C. 11-21.

3 Dauylbaev M.K., Mirzakulova A.E. Asymptotic behavior of solutions of singular integro-differential equations // Discontinuity, Nonlinearity and Complexity. - 2016. - Vol. 5. - No. 2. - P. 147-154.

4 Вишик М.И., Люстерник Л.А. Регулярное вырождение и пограничный слой для линейных дифференциальных уравнений с малым параметром // УМН. - 1957. - Т. 12. - № 5. - С. 3-122.

\author{
А.Е.Мирзакулова, Н.Атахан
}

\section{Мақалада жоғарғы туындыларының алдында кіші параметрі бар интегралды дифференциалдық теңдеулер үшін шекаралық есеп шешімінің аналитикалық формуласы}

\begin{abstract}
Мақала жоғарғы туындыларының алдында кіші параметрі бар $n+m$ ретті сызықты интегралдыдифференциалдық теңдеулер үшін бастапқы секірісті шекаралық есеп шешімінің аналитикалық формуласын зерттеуге арналған. Зерттеу жұмысында $n+m$ ретті біртекті дифференциалдық теңдеуінің іргелі шешімдер жүйесі алынған. Іргелі шешімдер жүйесінің көмегімен Коши функциясы және шекаралық функциялар құрылған. Мақалада Коши функциясы және шекаралық функцияларды қолданып, жоғарғы ретті сингулярлы ауыткыған интегралды-дифференциалдық теңдеу үшін қарастырылып отырған локалды шекаралық есеп шешімінің аналитикалық формуласы берілді.
\end{abstract}

А.Е.Мирзакулова, Н.Атахан

\section{Построение решения краевой задачи для интегро- дифференциальных уравнений с малым параметром при старших производных}

В статье исследованы аналитические формулы решений краевой задачи с начальным скачком для линейных интегро-дифференциальных уравнений $n+m$ порядка с малым параметром при старших производных. В работе построена фундаментальная система решений сингулярно возмущенного однородного дифференциального уравнения $n+m$ порядка. С помощью фундаментальных систем решений построены функции Коши и граничные функции. Используя функции Коши и граничные функции, получена явная аналитическая формула решений рассматриваемой локальной краевой задачи для сингулярно возмущенного интегро-дифференциального уравнения высшего порядка.

\section{References}

1 Kasymov K.A., Nurgabyl D.N., Uaisov A.B. Ukrainian Mathematical Journal, 2013, 5, p. 629-641. 2 Dauylbaev M.K., Mirzakulova A.E. Nonlinear oscillations, 2016, 19, 1, p. 11-21.

3 Dauylbaev M.K., Mirzakulova A.E. Discontinuity, Nonlinearity and Complexity, 2016, 5, 2, p. $147-154$.

4 Vishik M.I., Lyusternik L.A. UMN, 1957, 12, 5, p. 3-122. 